\title{
Effects of surface roughness on plastic strain localization in polycrystalline aggregates
}

\author{
Yoann Guilhem ${ }^{1, a}$, Stéphanie Basseville ${ }^{2}$, Henry Proudhon ${ }^{3}$ and Georges Cailletaud ${ }^{3}$ \\ ${ }^{1}$ Laboratoire de Mécanique et Technologie, ENS de Cachan, CNRS UMR 8535, 61 avenue du \\ Président Wilson, 94235 Cachan Cedex, France \\ ${ }^{2}$ LISV, Université de Versailles Saint-Quentin, 45 avenue des Etats-Unis, 78000 Versailles, France \\ ${ }^{3}$ Centre des Matériaux, MINES ParisTech, CNRS UMR 7633, BP. 87, 91003 Evry Cedex, France
}

\begin{abstract}
The surface state of mechanical components differs according to applied loadings. Industrial processes may produce specific features at the surface, such as roughness, local hardening, residual stresses or recrystallization. Under fatigue loading, all these parameters will affect the component lifetime, but in different manner. A better understanding of each surface state parameter, separately first and then all combined, will provide a better prediction of fatigue life. The study focuses on the effect of surface roughness. Crystal plasticity finite element computations have been carried out on threedimensional polycrystalline aggregates with different roughness levels. Local mechanical fields have been analyzed both at the surface and inside the bulk to highlight the competition between crystallography and roughness to impose localization patterns. As soon as surface roughness is strong enough, classical localization bands driven by grains orientation are replaced by localizations patterns driven by the local roughness topology. Nevertheless, this effect tends to decrease gradually under the surface, and it becomes usually negligible after the first layer of grains. The discussion allows us to characterize the influence of the surface state on the local mechanical fields.
\end{abstract}

\section{Introduction}

Fatigue crack initiation usually takes place at the component surface and is provoked by plastic strain localization along slip bands [1]. This is strongly driven by microstructural features, such as crystallographic orientation and grain boundaries. It has been observed at the macro scale that the surface state of a specimen can affect the fatigue life of a component [2]. This phenomenon has been recently investigated at the mesoscopic scale, using Finite Element computations of polycrystalline aggregates [3]. The aim of our numerical study is to quantify the impact of surface roughness on local mechanical fields, apart from other surface state parameters.

\footnotetext{
${ }^{a}$ Corresponding author: yoann.guilhem@ens-cachan.fr
}

This is an Open Access article distributed under the terms of the Creative Commons Attribution License 4.0, which permits unrestricted use, distribution, and reproduction in any medium, provided the original work is properly cited. 


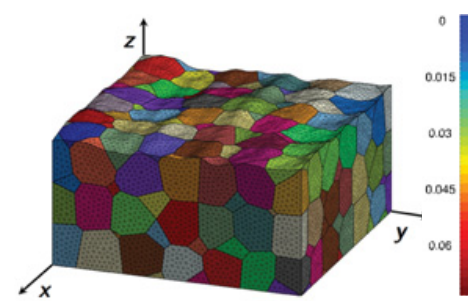

$\mathbf{a}$

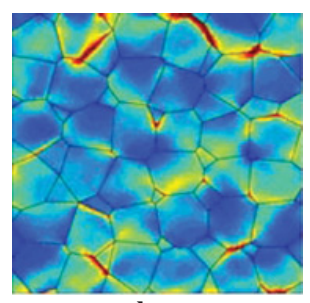

b

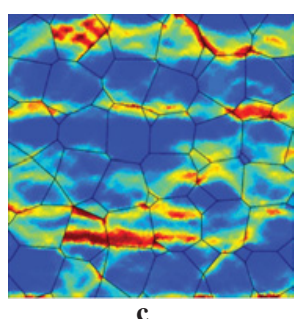

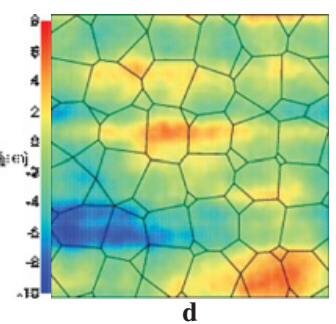

d

Figure 1. (a) Rough mesh. Plastic strain map for (b) flat case and (c) rough case. (d) Roughness map.

\section{Numerical model}

Méric-Cailletaud's crystal plasticity model is introduced as the local behaviour of each grain, considered as FCC single crystals. The material parameters have been identified on a 316L stainless steel [4]. A rough surface has been generated based on a 1D roughness profile of a brushed finished component and then applied on a 291 grains aggregate build by means of Voronoi tessellation (Fig. 1a). To investigate the effect of roughness intensity, various meshes have been produced by multiplying the roughness map by a factor $k_{r}$ between 0.1 and 1 . A 2 cycles loading is applied of $0.2 \%$ strain in uniaxial or biaxial mode. The results will be analysed on both the rough aggregate and the flat one, used as reference case.

\section{Results and discussion}

The sum of the accumulated plastic slip over all the slip systems is used to characterize plastic flow. Figure $1 \mathrm{~b}$ (respectively 1c) shows the plastic strain contour map at the free surface of the aggregate for the flat (respectively rough) case. This reveals strong changes in the plastic strain localization patterns. In the reference case, it initially appears as $45^{\circ}$ bands induced by crystallographic orientation whereas in the rough case, localization moves to the valleys of the rough surface. Grain orientation remains a major parameter because multiple zones still show strong discontinuities at grain boundaries. This observation highlights the competition between the localization due to crystallographic orientation and the one induced by the surface topology. As the roughness intensity factor decreases, these modifications are progressively vanishing, thus a critical roughness state exists from which the cause plastic strain localization switch from crystallography to roughness.

However, this effect is getting gradually weaker deep under the surface. Indeed after the first layer of grains, the difference becomes negligible. A statistical analysis based on each Gauss point values is used to determine the depth of the affected zone depending on the roughness intensity. The results provide a linear correlation between the two.

\section{Conclusion}

A crystal plasticity finite element analysis of flat and rough polycrystalline aggregates has been carried out to quantify the effect of the roughness on the local mechanical fields. A competition between crystallography and roughness in the localization process has been identified. The depth of the affected zone decreased linearly with roughness intensity. The next step is to study the influence of the grain size compared to the roughness parameters. 
FDMDII - JIP 2014

\section{References}

[1] B. Ma, C. Laird. Acta Mat. 37, 325 (1989)

[2] M. R. Bayoumi, A. K. Abdellatif. Eng. Fract. Mech. 51, 870 (1995)

[3] A. Le Pécheur, F. Curtit, M. Clavel, J.-M. Stéphan, C. Rey, P. Bompard. Int. J. Fatigue 45, 48 (2012)

[4] Y. Guilhem, S. Basseville, F. Curtit, J. Stéphan, G. Cailletaud. Comp. Mater. Sci. 70, 150 (2013) 